Artigo original

\title{
Intoxicação exógena por medicamentos na população jovem do Rio Grande do Sul
}

\author{
Exogenous drug intoxication among young people of Rio Grande do Sul
}

Elisa Inês Klinger ${ }^{1}$, Dionata Cristiano Schmidt ${ }^{1}$, Daniela Barbosa Lemos ${ }^{1}$, Luiza Pasa ${ }^{1}$, Lia Gonçalves Possuelo ${ }^{1}$, Andréia Rosane de Moura Valim ${ }^{1}$

${ }^{1}$ Universidade de Santa Cruz do Sul (UNISC), Santa Cruz do Sul, RS, Brasil.

\begin{abstract}
RESUMO
Justificativa e objetivos: a grande variedade de medicamentos disponíveis no mercado, trouxe importantes mudanças no aspecto de utilização de medicamentos, porém, surgem também, problemas relacionados com estes produtos, dentre estes, a intoxicação exógena, considerada um problema mundial no âmbito da saúde, principalmente entre mulheres. O objetivo do estudo foi analisar os dados notificados por intoxicação exógena por medicamentos entre adultos jovens do Rio Grande do Sul. Métodos: trata-se de um estudo descritivo ecológico sobre os casos de intoxicação exógena por medicamentos de indivíduos entre 20 e 39 anos de idade, do Rio Grande do Sul, notificados nos anos de 2011 a 2015, no Sistema de Informação de Agravos de Notificação (SINAN). Resultados: foram encontradas 1.278 notificações de intoxicações exógenas ocasionadas por medicamentos. Destas, $76,29 \%$ eram do sexo feminino, $85,21 \%$ eram brancos, $25,98 \%$ tinham o ensino fundamental incompleto e $85,52 \%$ residiam na zona urbana. Do total de casos notificados, destacam-se os 1.038 casos de tentativa de suicídio $(81,28 \%)$, seguidos dos 68 casos por automedicação $(5,32 \%)$. Conclusões: a intoxicação exógena por medicamentos entre adultos jovens no Rio Grande do Sul é grave e preocupante, apontando a importância de medidas educativas de prevenção e promoção da saúde nessa população.
\end{abstract}

DESCRITORES: Medicamentos. Intoxicação. Adulto jovem.

\section{ABSTRACT}

Background and objective: the wide variety of drugs available in the market, brought important changes in the aspect of drug use, however, there are also problems related to these products, among these, exogenous intoxication, considered a world wide problem in the health sector, especially among women. The aim of the study was to analyze the data reported by exogenous drug intoxication among young adults of Rio Grande do Sul. Methods: it is an ecological descriptive study of cases of exogenous drug intoxication of individuals between 2039 years of age, from Rio Grande do Sul, reported in the years 2011-2015, at Information System for Notifiable Diseases (SINAN). Results: 1,278 notifications of exogenous intoxication caused by drugs were found. Of these, $76.29 \%$ of the cases were female, $85.21 \%$ were white, $25.98 \%$ had incomplete primary education and $85.52 \%$ lived in urban areas. Of the reported cases, it highlights the 1,038 cases of attempted suicide ( $81.22 \%)$, followed by 68 cases of self-medication $(5.32 \%)$. Conclusions: the exogenous drug intoxication among young adults 
in Rio Grande do Sul is serious and worrying, indicating the importance of educational measures of prevention and health promotion in this population.

KEYWORDS: Pharmaceutical Preparations. Poisoning. Young Adult.

\section{INTRODUÇÃO}

A consequência da interação entre agente tóxico e organismo se define como intoxicação exógena, que ocorre quando o agente tóxico rompe o equilíbrio orgânico, alterando funções bioquímicas e fisiológicas. A toxicidade compreende o potencial, em maior ou menor grau, do surgimento de um estado patológico como resposta. ${ }^{1}$ Conforme o Sistema Nacional de Informações Tóxico - Farmacológicas (SINITOX), os medicamentos caracterizam-se como um dos principais agentes causadores de intoxicação em seres humanos no Brasil, estando emprimeiro lugar nas estatísticas deste sistema, sendo que nos últimos cinco anos, foram registrados no Brasil, aproximadamente 60 mil internações por intoxicação medicamentosa por meio desse sistema. ${ }^{2}$

A intoxicação exógena tem sido considerada um problema mundial no âmbito da saúde e o uso indevido de medicamentos têm aumentado consideravelmente, trazendo consigo a resistência bacteriana, reações de hipersensibilidade, dependência química, sangramento digestivo e também predisposição ao surgimento de neoplasias. ${ }^{1,3} \mathrm{O}$ consumo da medicação teve um aumento considerável nos últimos anos, crescimento este, que se torna expressivo na população mundial, inclusive em países desenvolvidos. ${ }^{4}$ Este consumo elevado de medicamentos já é considerado um hábito no cotidiano da população, podendo estar relacionado ao uso abusivo, bem como a aspectos individuais e sociais, ou também com a tentativa de suicídio em alguns casos. ${ }^{5}$

A indústria farmacêutica tem crescido muito nos últimos anos, desenvolvendo novos produtos e realizando importantes mudanças no aspecto de utilização de medicamentos em esfera mundial. Com a grande variedade de medicamentos disponíveis no mercado brasileiro, surgem também, problemas relacionados com estes produtos. ${ }^{6}$ A intoxicação exógena por medicamentos, é um destes problemas, representando uma das formas mais frequentes no Brasil, sendo as mulheres um dos grupos mais vulneráveis, principalmente quanto aos casos de tentativa de suicídio, o que torna cada vez mais importante a realização de estudos estatísticos analisando o perfil desta população, bem como, os aspectos de relevância na área. ${ }^{7}$ Diante do exposto, este estudo tem por objetivo analisar os dados notificados por intoxicação exógena por medicamentos entre adultos jovens do Rio Grande do Sul entre os anos de 2011 a 2015. 


\section{MÉTODOS}

Trata-se de um estudo descritivo ecológico, com o enfoque nos casos de intoxicação exógena por medicamentos na população de adultos jovens de 20 a 39 anos de idade, notificados no Rio Grande do Sul, nos anos de 2011 a 2015, no Sistema de Informações de Agravos e Notificações (SINAN) do Departamento de Informática do SUS (DATASUS) do Ministério da Saúde. Foram avaliadas as variáveis: sexo, raça, escolaridade, localização, município de residência e circunstância da intoxicação. As tabelas foram construídas através do software Excel versão 2007 e o mapa através do programa Tabwin do DATASUS, considerando o número absoluto de casos de intoxicação exógena por medicamentos, notificados nos anos de 2011 a 2015, por município de residência, estratificados por sexo e apenas a faixa etária de 2039 anos de idade, utilizando o mapa do Rio Grande do Sul, apontando os casos nos seus respectivos municípios. Quanto às considerações éticas, as informações utilizadas são de livre acesso e não envolvem conflitos de interesse.

\section{RESULTADOS}

Foram encontrados um total de 7.767 notificações de intoxicações exógenas no Estado do Rio Grande do Sul, segundo município de residência, abrangendo todos os agentes tóxicos e faixas etárias. Considerando a faixa etária com o maior número de notificações, a dos 20 aos 39 anos, foram notificados 3.787 casos (48,76\%), dos quais 1.278 casos eram por medicamentos, correspondendo a $33,75 \%$ dos casos notificados por intoxicação exógena dentre adultos jovens. Dos 1.278 casos de intoxicação por medicamentos, 76,29\% eram do sexo feminino, $85,21 \%$ eram de pessoas brancas, $25,98 \%$ tinham o ensino fundamental incompleto e $85,52 \%$ residiam na zona urbana de seus municípios (Tabela 1).

Tabela 1- Características descritivas dos sujeitos notificados com intoxicação exógena por medicamentos na faixa etária de 20-39 anos.

\begin{tabular}{lccc}
\hline & $\begin{array}{c}\text { Masculino } \\
\mathbf{n}(\boldsymbol{\%})\end{array}$ & $\begin{array}{c}\text { Feminino } \\
\mathbf{n}(\boldsymbol{\%})\end{array}$ & $\begin{array}{c}\text { Total } \\
\mathbf{n}(\boldsymbol{\%})\end{array}$ \\
\hline Raça & & & $1.089(85,21)$ \\
Branco & $255(19,95)$ & $834(65,26)$ & $60(4,69)$ \\
Preto & $18(1,41)$ & $42(3,29)$ & $1(0,08)$ \\
Amarelo & $1(0,08)$ & $0(0,00)$ & $102(7,98)$ \\
Pardo & $22(1,72)$ & $80(6,26)$ & $26(2,03)$ \\
Não informado & $7(0,55)$ & $19(1,49)$ & $5(0,39)$ \\
Escolaridade & & & $332(25,98)$ \\
Analfabetos & $4(0,31)$ & $1(0,08)$ & $122(9,55)$ \\
Ensino fundamental incompleto & $66(5,16)$ & $266(20,81)$ & $133(10,41)$ \\
Ensino fundamental completo & $32(2,50)$ & $90(7,04)$ & $278(21,75)$ \\
Ensino médio incompleto & $31(2,43)$ & $102(7,98)$ & $221(17,29)$ \\
Ensino médio completo & $57(4,46)$ & &
\end{tabular}


Ensino superior incompleto

Ensino superior completo

Não informado

Localização

Urbana

Rural

Periurbana

Não informado

Total

$12(0,94)$
$6(0,47)$
$95(7,43)$
$254(19,87)$
$31(2,42)$
$12(0,94)$
$6(0,47)$
$303(23,71)$

$41(3,21)$

$18(1,41)$

$236(18,47)$

$839(65,65)$

$91(7,12)$

$32(2,50)$

$13(1,02)$

$975(76,29)$
$53(4,15)$

$24(1,88)$

$331(25,90)$

$1.093(85,52)$

$122(9,55)$

$44(3,44)$

$19(1,49)$

$1.278(100)$

Na Figura 1 estão representados os casos de intoxicação exógena por medicamentos notificados por município, estratificados por sexo feminino (1a) masculino (1b), na faixa etária de 20-39 anos. Os municípios com maior número de casos de notificação de intoxicação por medicamentos são: Marau, Passo Fundo, Teutônia, Santa Maria, Santiago e Cachoeira do Sul, respectivamente. Quando avaliada a circunstância da intoxicação medicamentosa, foi desconsiderado um caso notificado por aborto no sexo masculino, por incompatibilidade da informação, considerando 1.277 casos; dentre estes, destacam-se os 1.038 casos notificados por tentativa de suicídio, correspondendo a $81,28 \%$ dos casos, sendo que destes, as mulheres foram as que mais tentaram o suicídio por medicamentos $(62,73 \%)$. A segunda circunstância que se destacou, foi a automedicação, com 68 casos (5,32\%), como é possível visualizar na Tabela 2. 
Figura 1 -Número absoluto de casos de intoxicação exógena por medicamentos notificados por município, estratificados por sexo, na faixa etária de 20-39 anos.
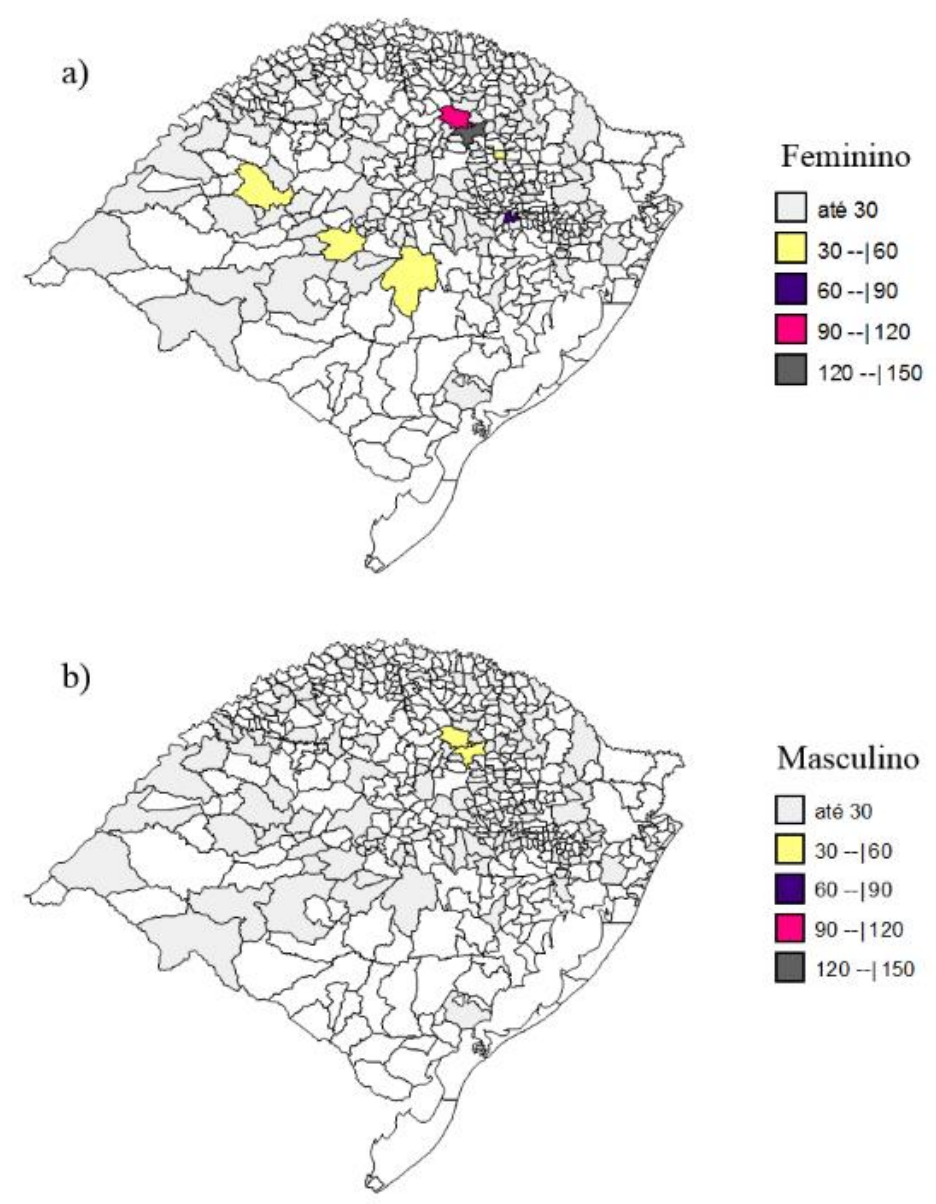

Tabela 2 -Casos de intoxicação exógena notificados por circunstância

\begin{tabular}{lccc}
\hline & $\begin{array}{c}\text { Masculino } \\
\mathbf{n}(\boldsymbol{\%})\end{array}$ & $\begin{array}{c}\text { Feminino } \\
\mathbf{n}(\boldsymbol{\%})\end{array}$ & $\begin{array}{c}\text { Total } \\
\mathbf{n}(\boldsymbol{\%})\end{array}$ \\
\hline Circunstância & & & \\
Uso habitual & $7(0,55)$ & $22(1,72)$ & $29(2,27)$ \\
Acidental & $7(0,55)$ & $11(0,86)$ & $18(1,41)$ \\
Uso terapêutico & $3(0,23)$ & $2(0,16)$ & $5(0,39)$ \\
Erro de administração & $1(0,08)$ & $8(0,63)$ & $9(0,70)$ \\
Automedicação & $19(1,49)$ & $49(3,84)$ & $68(5,32)$ \\
Abuso & $13(1,02)$ & $21(1,64)$ & $34(2,66)$ \\
Ingestão de alimentos & $2(0,16)$ & $7(0,55)$ & $9(0,70)$ \\
Tentativa de suicídio & $237(18,56)$ & $801(62,73)$ & $1038(81,28)$ \\
Tentativa de aborto & $0(0,00)$ & $14(1,10)$ & $14(1,10)$ \\
Violência/homicídio & $4(0,31)$ & $9(0,70)$ & $13(1,02)$ \\
Ignorado & $8(0,63)$ & $28(2,19)$ & $36(2,82)$ \\
Outras & $1(0,08)$ & $3(0,23)$ & $4(0,31)$ \\
\hline
\end{tabular}




\section{DISCUSSÃO}

Neste estudo, as mulheres destacam-se por representarem a maior parte dos casos notificados por intoxicação exógena. Teles et al. (2013) observaram no município de Feira de Santana, na Bahia, que o sexo feminino foi o responsável pelo maior número de registros de intoxicação medicamentosa. ${ }^{8}$ Quanto ao grau de escolaridade, foi alto o índice de notificações não informadas $(25,90 \%)$, contudo grande parte dos indivíduos tinha ensino médio completo $(21,75 \%)$ ou não tinham o ensino fundamental completo $(25,98 \%)$, diferente do estudo citado acima que mostrou que a maioria dos acometidos tinha como escolaridade o 'ensino fundamental completo' $(27,4 \%, \mathrm{n}=17)$.

Os casos de intoxicação exógena por medicamentos, notificados entre 2011 e 2015 , foram mais expressivos entre as mulheres. A tentativa de suicídio foi a principal circunstância associada a intoxicações medicamentosas nos jovens adultos avaliados no Rio Grande do Sul, principalmente deste sexo. Resultados semelhantes foram encontrados em um estudo realizado no Canadá, com jovens com idades compreendidas entre os 18 e os 36 anos, sendo que aproximadamente um terço dos adultos jovens que receberam atendimento médico por tentativa de suicídio, além de serem do sexo feminino, eram universitários, e metade destes jovens estavam deprimidos no momento da tentativa, estando mais propensos a usar o envenenamento em comparação com métodos de suicídio mais violentos. ${ }^{9}$

A letalidade da forma de suicídio utilizada, não está diretamente relacionada à intenção de morte em si, mas com a aceitação social que teria o sexo como base para a escolha do método suicida. Assim, o fato das mulheres optarem pelo uso de medicamentos como meio de suicídio, seria socialmente mais aceita, em comparação com os homens, dos quais seria mais presumível um meio mais violento, como enforcamento ou uso de arma de fogo, por exemplo. Desse modo, estando os medicamentos disponíveis a ambos os sexos, a escolha envolve, em certo grau, além da disponibilidade ao método, a aceitabilidade social. ${ }^{10}$

Quanto aos casos de automedicação, embora não sejam muito expressivos, é a segunda circunstância mais notificada de intoxicação por medicamentos entre 2011 e 2015, na faixa etária avaliada neste estudo. Isso é preocupante, pois o indivíduo expõe sua saúde ao se automedicar sem diagnóstico e prescrição médica. Deste modo, a automedicação constitui um fator de risco relacionado com intoxicação por medicamentos, necessitando de aprofundamento, considerando que nem todos medicamentos precisam de receituário médico para a aquisição. A automedicação pode estar relacionada com outras ocorrências de intoxicação, tais como: uso acidental, tentativa de suicídio e uso excessivo de medicamentos 
sem prescrição, devido ao livre acesso ou pelas aquisições repetidas após prescrição inicial. ${ }^{11}$ Segundo Nebeker, Barach, Samore (2004), distintas razões levam o indivíduo a se automedicar, dentre elas pode-se citar a dificuldade de acesso aos serviços básicos de saúde, bem como a profissionais responsáveis prescritores de medicamento, assim como a falta de fiscalização de órgãos públicos competentes e os distintos interesses comerciais que levam a população a isso. ${ }^{12}$ Essas situações levaram ao aumento da automedicação em diversas regiões do mundo, inclusive no Brasil, de forma mais marcante nas regiões menos favorecidas. ${ }^{4}$

Dentre as limitações deste estudo, está o elevado número de informações notificados como 'ignorado/em branco', especialmente, para as variáveis 'escolaridade' e 'circunstância', tornando-se um empecilho para uma análise fidedigna desses dados. Esta situação pode estar relacionada a problemas de registro, como descuido do profissional responsável pelo preenchimento ou desconhecimento de quem informa, sendo necessário melhorar a qualidade dos dados registrados no sistema, podendo os casos subnotificados, estarem relacionados a questões culturais/religiosas e legais. ${ }^{10}$

Concluímos que a intoxicação por medicamentos pode ser considerada um problema de saúde pública, que torna necessário priorizar ações educativas, intersetoriais e interdisciplinares de forma continuada. Estas ações devem ter a finalidade de garantir o uso correto e racional de medicamentos com o objetivo de reduzir, especialmente, os casos de tentativas de suicídio em adultos jovens, principalmente em indivíduos do sexo feminino, que são mais predispostos a este tipo de agravo. O conhecimento dos fatores específicos, associado a tentativas de suicídio em jovens, pode auxiliar a informar e orientar, no intuito de prevenir ou reduzir as tentativas de suicídio em ambientes acadêmicos e comunitários. No caso de automedicação e outras formas de intoxicação exógena, é importante limitar o acesso a substâncias perigosas ou grandes quantidades de medicamentos.

\section{REFERÊNCIAS}

1. Zambolim CM,Oliveira TP, HoffmannAN, et al. Perfil das intoxicações exógenas em um hospital universitário. RevMéd Minas Gerais 2008; 18(1): 5-10.

2. SINITOX. Sistema Nacional de Informações Tóxico-Farmacológicas. Rio de Janeiro. 2013 [acesso em 22 junho 2016]. Disponível em: http://www.fiocruz.br/sinitox.

3. Galato D, Madalena J, Pereira GB. Automedicação em estudantes universitários: a influência da área de formação. Ciênc \& Saúde Coletiva 2012;17(12):3323-3330. doi: http://dx.doi.org/10.1590/S1413-81232012001200017. 
4. Vosgerau ZS; Soares DA, Souza RKT. Automedicação entre adultos na área de abrangência de uma Unidade Saúde da Família. LatAm J Pharm 2008;27(6):831-8.

5. Richetti GP, De Pinho Alves Filho J. Automedicação no Ensino de Química: uma proposta interdisciplinar para o Ensino Médio. Educación Química 2014:25(E1), 203 209.doi: http://dx.doi.org/10.1016/S0187-893X(14)70559-2

6. Margonato FB,Thomson Z,Paoliello MMB. Determinantes nas intoxicações medicamentosas agudas na zona urbana de um município do Sul do Brasil. Cad Saúde Pública 2008; 24(2):333-341. doi: http://dx.doi.org/10.1590/S0102$311 X 2008000200012$

7. Bortoletto ME,Bochner R.Impacto dos medicamentos nas intoxicações humanas no Brasil. Cad Saúde Pública1999; 15(4):859-869.doi: http://www.arca.fiocruz.br/handle/icict/460

8. Teles AS, Oliveira RFA, Coelho TCB, et al. Papel dos medicamentos nas intoxicações causadas por agentes químicos em município da Bahia, no período de 2007 a 2010. RevCiêncFarm Básica Apl 2013;34(2):281-288.

9. Rahme E, Low NCP, Lamarre S, et al.Attempted Suicide Among Students and Young Adults in Montreal, Quebec, Canada: A Retrospective Cross-Sectional Study of Hospitalized and Nonhospitalized Suicide Attempts Based on Chart Review. PrimCare Companion CNS Disord 2015;17(5). doi:10.4088/PCC.15m01806.

10. Santos SA, LegayFL, Lovisi GM.Substâncias tóxicas e tentativas e suicídios: considerações sobre acesso e medidas restritivas. Cad Saúde Colet2013;21(1):5361.doi: http://dx.doi.org/10.1590/S1414-462X2013000100009

11. Gandolfi E, Andrade MGG. Eventos toxicológicos relacionados a medicamentos no Estado de São Paulo. RevSaúde Pública 2006;40(6):1056-64.doi: http://dx.doi.org/10.1590/S0034-89102006000700014

12. Nebeker JR, Barach P, Samore MH. Clarifying adverse drug events: A clinician's guide to terminology, documentation, and reporting. Ann Intern Med 2004:140(10):795801.doi:10.7326/0003-4819-140-10-200405180-00017 